\title{
TEATRO FANTÁSTICO A PRINCIPIOS DE SIGLO XX: UNA INTRODUCCIÓN AL CONTEXTO Y A LOS TEXTOS DRAMÁTICOS DE EDUARDO CALSAMIGLIA
}

Jessie Zúñiga Bustamante

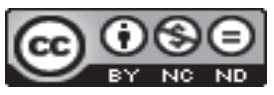

Esta obra está bajo una licencia Creative Commons Reconocimiento-No Comercial-Sin Obra Derivada 



\title{
TEATRO FANTÁSTICO A PRINCIPIOS DE SIGLO XX: UNA INTRODUCCIÓN AL CONTEXTO Y A LOS TEXTOS DRAMÁTICOS DE EDUARDO CALSAMIGLIA
}

\author{
FANTASTIC THEATER IN THE EARLY 20 ${ }^{\text {TH }}$ CENTURY:AN \\ INTRODUCTION TO THE CONTEXT AND TO THE DRAMATIC TEXTS \\ BY EDUARDO CALSAMIGLIA
}

Jessie Zúñiga Bustamante

\begin{abstract}
RESUMEN
Los textos dramáticos de Eduardo Calsamiglia representan una ruptura con el modelo estético e ideológico de su época. La presencia de ambientes misteriosos, sobrenaturales o fantásticos como parte de la trama lo acerca más a la renovación teatral de fines de siglo XIX en Europa, especialmente en España, con el denominado "teatro de ensueño". Asimismo, hay en ellos una preocupación filosófica sobre una particular noción del mal correspondiente al auge del positivismo y la Modernidad. Es por eso que sus textos deben ser reevaluados y reconocidos por su valor literario, así como por el aporte que significan en el marco de la ruptura estética del teatro Occidental de finales del siglo XIX y principios del XX.

Palabras clave: literatura fantástica, teatro costarricense, teatro de ensueño, finisecular, Casalmiglia-Eduardo.
\end{abstract}

\section{ABSTRACT}

Eduardo Calsamiglia's plays represent a break with the aesthetic and ideological model of his time. The presence of mysterious, supernatural or fantastic elements in his plots tie them more to the theater renovation that took place in the late nineteenth century in Europe, especially in Spain, the so-called "teatro del ensueño". There is also a philosophical concern on a particular notion of evil which coincides with the rise of positivism and Modernity. That's why his texts should be reassessed and recognized for their literary value, as well as the contribution they hold in the context of aesthetic rupture with the Western theater of the late nineteenth and early twentieth centuries. Key words: theater, fantastic, dream theater, finisecul, Casalmiglia-Eduardo.

La tradición literaria costarricense rara vez asocia la primera mitad del siglo pasado con lo fantástico. Tanto más si la ecuación involucra al teatro. La literatura de principios de siglo XX se suele -comúnmente- afiliar con el discurso fundacional de las letras y la nacionalidad o con las costumbres, versus una corriente "europeizada" que deja de lado el contexto y la problemática locales. Poco se menciona en la crítica a autores y textos que escapen a esa dicotomía.

Bach. Jessie Zúñiga Bustamante. Universidad de Costa Rica. Estudiante de la Maestría en Literatura Latinoamericana. Costa Rica.

Correo electrónico: jessiezbustamante@gmail.com

Recepción: 20- 12- 2013

Aceptación: 15- 03- 2014 
El teatro de Eduardo Calsamiglia es un ejemplo de ello. En sus textos hay una serie de elementos que coinciden con los de la renovación teatral de fin de siglo XIX en Europa. Se trata de obras dramáticas en las que se presentan atmósferas misteriosas, angustiantes, o bien, en las que lo sobrenatural es parte de la trama. Asimismo, se caracterizan por la confluencia de rasgos tanto narrativos como dramáticos en la elaboración de lo fantástico y hay en ellas una preocupación filosófica sobre una particular noción del mal correspondiente al auge del positivismo y la Modernidad. Es por eso que sus textos deben ser reevaluados y reconocidos por su valor literario, así como por el aporte que significan en el marco de la ruptura estética del teatro Occidental de finales del siglo XIX y principios del XX.

Eduardo Calsamiglia nació en San José (de padre catalán y madre costarricense) en 1880. A lo largo de su vida desempeñó muy diversas ocupaciones; fue militar, diplomático, poeta, profesor de gimnasia y astrónomo aficionado (Díaz, 2005). Esto último resulta muy importante para el tema que nos ocupa, pues es muy posible que esta visión de los fenómenos de la naturaleza y del conocimiento científico de su época lo haya llevado a plasmar sus ideas en sus textos y, de manera particular, en su teatro.

A pesar de su corta vida -Calsamiglia murió a la edad de 38 años- su contribución a la literatura costarricense es valiosísima -sobre todo como pionero del teatro- y abarca varios géneros; su primera publicación data de 1898, con Versos y cuentos y, por ejemplo, en 1906 escribió un tratado llamado Táctica de infantería para el ejército de aquel entonces. En 1917, fue ascendido a General y Tinoco lo nombró Encargado de Negocios-Ministro Plenipotenciario ante el Gobierno de Guatemala. Allí, un año más tarde, murió a causa de la muy conocida epidemia de influenza que azotó en dicha época.

De particular importancia resultan los viajes que Calsamiglia hace a España entre 1885 y 1898. Luis Gustavo Lobo-Bejarano, en el estudio introductorio a su Obra literaria (2006), menciona que el primero de ellos lo hace en 1885 (a los 5 años); el segundo, en 1892, de los que regresó un año después; y un tercer viaje en 1893 del cual regresaría hasta 1898, cinco años durante los cuales -de acuerdo con Lobo-Bejarano (2006) y Bonilla (1981)- realizó estudios de literatura en Barcelona.

Como vemos, Bonilla no se equivoca en sus datos pero al viaje que se refiere es evidentemente el último, pues solamente residiendo más de un año en un país es posible hacer estudios de una materia como la literatura. (Quesada-Soto y Lobo-Bejarano, 2006, p. 5)

Este dato nos permite afirmar que Calsamiglia no solo está al tanto de las corrientes literarias de su época gracias a las puestas en escena que se realizaban en San José, sino que además estuvo en contacto directo con ellas en su estancia en Barcelona y sus visitas a Londres y París (Quesada-Soto y Lobo-Bejarano, 2006, p. 10), especialmente en una época de grandes cambios en cuanto al teatro se refiere.

Otro dato interesante y que, además de curioso, consideramos clave para comprender su posición crítica y estética, es el que aporta Ronal Eduardo Díaz Bolaños en su artículo "«Los costarricenses no han dejado de admirar las magnificencias del cielo»: la evolución de las ideas astronómicas en Costa Rica (1814-1910)" (Díaz, 2005), en el que nos menciona a Calsamiglia como uno de los intelectuales de la época más interesados en los conocimientos científicos y los adelantos teóricos, frente a la 'supersticiosa' y 'religiosa' sociedad costarricense de fin de siglo. Comenta Díaz que Calsamiglia poseía un telescopio en el fortín de la ciudad de Heredia, para hacer sus propias observaciones y estudios astronómicos. De acuerdo con Díaz (2005), el 
paso del famoso cometa Halley, en 1910 por cielo nacional, causó una ebullición a todos los niveles, desde el científico de corte positivista -acorde con la herencia de la Ilustración y la Edad Moderna, al cual se une Calsamiglia- hasta la superchería más chabacana y amarillista de medios de comunicación y de toda clase de discursos religiosos y fatalistas a los que tenía acceso la mayor parte de la población. Esto preocupaba a los intelectuales y científicos más serios, estudiosos de la astronomía, quienes entraron en acalorada polémica con las alas más tradicionales y conservadoras de la vida científica del país, en una lucha por educar e informar al público sobre los fenómenos de la naturaleza y cómo el paso de este cometa no significaba el fin del mundo según afirmaban las ideas y supersticiones que circulaban ampliamente en los medios. Esto llevó a la publicación de folletines y otros textos de evidente corte didáctico que pretendían ser una guía para comprender, fuera de toda posición conservadora, religiosa y poco formal, lo que ocurría.

Esta información se confirma en el prólogo que hace Joaquín Vargas-Coto a la edición del drama póstumo Bronces de antaño, de 1919, en el que nos dice:

\footnotetext{
Autor de dramas y zarzuelas, escritor de tácticas y ordenanzas militares, también cultivó la filosofía y la ciencia, sabiendo de astronomía, bastante de medicina y deleitándose en la gimnasia espiritual que consiste andar por los campos metafísicos y desconocidos... De ahí que fuera uno de los espíritus más dilectos y mejor cultivados de nuestro país, así como uno de los intelectuales de más mérito. (Quesada-Soto y Lobo-Bejarano, 2006, p. 465)
}

Este dato es sumamente valioso, tomando en cuenta los textos literarios de Calsamiglia, pues en ellos es evidente que el tema de la ciencia que supera lo "sobrenatural", "lo extraño", la "superstición" y la doxa, es la esencia que configura el contenido (Trancón, 2006). Así, por ejemplo, en El combate, en la que un médico logra vencer su deseo de venganza y sus emociones, en pro de su profesión y el bien de la ciencia, o en ¡Él! en la que, como se verá más adelante, lo fantástico da paso a la explicación racional y a la resolución lógica de los conflictos.

\section{El teatro a fines del siglo XIX y principios del XX: panorama general}

De acuerdo con múltiples estudios (Quesada-Soto, 1995; Rojas y Ovares, 1995; etc.) la literatura costarricense se consolida en un período cuyos procesos socioeconómicos llevan a una acelerada modernización del país. La inserción de Costa Rica en el modelo capitalista europeo por medio de la exportación de café a Europa, propició una transformación urbana sin precedentes y, en este clima de cambios, Costa Rica experimentó un auge de los espectáculos que contribuyó indudablemente con el desarrollo de la dramaturgia nacional.

La investigadora Flora Ovares (2008) señala los diversos cambios en la sociedad de finales del siglo XIX, como la inauguración de los teatros (Variedades en 1889 y Nacional en 1897), del tranvía (en 1899), así como la creciente urbanización de la ciudad capital. Sumado a ello, menciona cómo estas variables inciden en el auge de los espectáculos, que se extendió hasta 1914 aproximadamente, gracias a las puestas en escena de compañías mexicanas y españolas (de ópera, zarzuela y drama). Tanto Ovares como Bonilla (1981) apuntan hacia la relevancia de estas compañías que representaban, entre otras, obras de José de Echegaray "el público se nutría de obras complicadas y declamatorias como las de Echegaray que representaban las compañías españolas" (Bonilla, 1981, p. 201) y Benavente: "la presencia de las compañías de ópera, zarzuela y drama que permanecían por largas temporadas en San José hablan de una necesidad de esparcimiento, y consumo de productos culturales" (Ovares, 2008, p. 57). 
De la mano de los espectáculos, crece también la circulación de revistas literarias que dan a conocer a grandes autores como Darío, Gutiérrez Nájera y Del Casal. Pero estas revistas no solamente son importantes por la difusión de los modernistas, sino porque un componente fundamental en ellas fueron las traducciones de escritores europeos de gran influencia para los nuevos escritores, como el caso del francés Guy de Maupassant.

\begin{abstract}
Se publican páginas de Goethe, D’Annunzio, Catulle Méndes, Mallarmé, Anatole France, Maupassant, Heredia, Blanco Fombona, Herrera y Reissig, Gutiérrez Nájera, Amado Nervo y muchísimos otros.

Las traducciones suelen ser obra de los escritores nacionales, que se preocupan de dar a conocer la poesía europea. Así, en 1903, Alejandro Alvarado y Fabio Baudrit vierten al castellano once narraciones de cuentistas franceses, como Maupassant, France y Marcel de Prevost y las publican bajo el título de Piedras preciosas. (Ovares, 2008, p. 58)
\end{abstract}

Abelardo Bonilla (1981) en su famosa Historia de la literatura costarricense hace un brevísimo recorrido por la dramaturgia desde la época colonial hasta el siglo XIX. Al igual que otros críticos, señala 1900 como momento inaugural del teatro en Costa Rica. Curiosamente, incluye Bonilla el prólogo con que se publicó Magdalena, la obra de Fernández Guardia que, en opinión del crítico, es "la primera obra dramática de carácter nacional" (Bonilla, 1981, p. 201), en dicho prólogo Fernández Guardia hace mención a varios puntos sumamente curiosos. En primer lugar, empieza refiriéndose a la "severidad" que ha caracterizado la recepción de su obra, de lo que podemos concluir que el "cuadro de costumbres costarricenses exacto y verdadero" (Bonilla, 1981, p. 201), cuyo personaje antagónico describe así: "sus ideas son exóticas, sus aspiraciones atrevidas; tiene la cabeza llena de literatura malsana" (Bonilla, 1981, p. 201), no fue del agrado del público de la época. En segundo lugar, se refiere al "especial" gusto de ese público de "paladares viciados a salsas más complejas" del que nos dice que tiene una "inclinación manifiesta a los efectos escénicos de brocha gorda y a su declamación altisonante” (Bonilla, 1981, p. 202). Y por último, cierra su prólogo con una suerte de reclamo que a nosotros nos brinda información muy valiosa sobre el tipo de teatro representado en Costa Rica y que, al parecer, dominaba el gusto del público en el país:

Como hemos tenido que contentarnos siempre con un arte extranjero, llámese francés o español, que sólo (sic) pone ante nuestros ojos costumbres, tipos y caracteres exóticos, hemos llegado a forjarnos una idea especialísima del teatro, que para el caso queda convertido en una especie de fantasmagoría, tanto más interesante cuanto más rara e inverosímil. De ahí que sea poco menos que imposible, hoy por hoy, interesar al público en el teatro con cosas nuestras y tipos nuestros. (Bonilla, 1981, p. 202)

Mientras, en España, de acuerdo con Jesús Rubio Jiménez (1989) en el siglo XIX se produjeron grandes avances en la representación escénica, esto es, en la técnica de escenificación y, al mismo tiempo, hay una creciente insatisfacción tanto de los artistas como del público con las representaciones en ese entonces vigentes. En ese contexto, se consolida un teatro "fantástico", mejor conocido como "simbolista" o "de ensueño" y se trata de textos que muestran una atmósfera alejada de la común realidad del espectador y lo envuelven en ambientes misteriosos y simbólicos. Estos textos representan, en palabras de Rubio "un modo particular de entender el teatro no como una imitación verista de la realidad inmediata, sino como construcción de mundos ficticios" (Rubio, 1989, p. 218).

Los temas y recursos de este teatro tan particular se nutren de la herencia de los grandes fundadores de la literatura de horror y de misterio, del mundo literario más fantástico de Shakespeare como el de Sueño de una noche de verano o Macbeth, de la literatura macabra y simbolista, de la representación compleja y simbólica del Fausto de Goethe, y por qué no, de la influencia de las corrientes romántica y posromántica que, de acuerdo con 
el teórico Bernard Sichère, "convocan tan profusamente las figuras de lo maléfico y de la maldición... para darle sentido partiendo de una subjetividad nueva que descubre en sí misma (no solamente en el exterior) la virtualidad de un momento de horror, de deseo extremo y de goce sin nombre" (Sichère, 1995, p. 188).

\section{Lo fantástico, un territorio difuso y conflictivo}

Matteo de Beni en su estudio "Para una historia de la palabra "fantástico" en España" (2010) hace un amplio recorrido por la lexicografía española, así como por el uso del término en el lenguaje dramatúrgico desde, más o menos, la mitad del siglo XIX hasta principios del XX. De Beni registra las diversas acepciones del vocablo fantástico entre las que destaca la de "fingido" o "imaginado" -también en italiano, francés e inglés-, pasando por "imaginario", "quimérico", "falso", "preternatural", "visión”, "irreal” hasta connotaciones menos comunes como "inmaterial", "intangible", "incorpóreo" (De Beni, 2010, pp. 47-49). Este investigador señala que se registran usos del término "fantástico" ya desde el siglo XIII en España, con la Primera Partida alfonsí (de acuerdo con el CORDE, Corpus diacrónico del español) y en el siglo XIV en Francia el adjetivo "fantastique". En el ámbito literario, su inclusión sería posterior, cuando Walter Scott en 1927 utiliza "fantastic mode" para definir los cuentos de Hoffmann, que se tradujo al francés como "contes fantastiques" y de esta forma pasan a España entre 1830 y 1831 (De Beni, 2010, p. 51).

En cuanto al teatro finisecular, propiamente, De Beni afirma que abundan los subtítulos que subrayan el carácter fantástico de las obras en "combinaciones y fórmulas inéditas e inesperadas" (De Beni, 2010, p. 53) en las que apunta más bien a una suerte de "práctica escénica" (De Beni, 2010, p. 53) que persigue un efecto sorpresivo o asombroso y, en otros casos, es utilizada como "sinónimo de "raro", "extraño" o "exótico"” (De Beni, 2010, p. 54), pero que en general poco o nada tienen en común con la definición de lo fantástico desarrollada en el siglo XX.

En efecto, a partir del la segunda mitad del siglo XX lo fantástico literario se sistematiza en una serie de teorías encabezadas por la crítica literaria francesa (Todorov, 2006; Vax, 1973; Callois, 1965, etc.) y, posteriormente, en toda hispanoamérica hasta los más recientes aportes de Jaime Alazraki (2001), Rosalba Campra (2008), Víctor Bravo (1985) y David Roas (2001), entre otros, quienes plantean una especificidad de lo fantástico como una ruptura de las leyes que gobiernan lo cotidiano, una problematización de la lógica o de la nociones de "realidad" y hasta de "sujeto", a partir de la desestabilización del pensamiento moderno, racional, dieciochesco.

En resumen, tendríamos al menos tres momentos clave en el desarrollo del término fantástico, a saber: el más apegado a la etimología grecolatina (fantastikós, fantasticusimaginarius), el referido a atmósferas enrarecidas, misteriosas y simbólicas y la presencia de elementos celestiales o bien malignos y sobrenaturales (asociado al teatro del ensueño) y, por último, el que constituye una figura liminar, como momento de vacilación (Todorov, 2006), de ruptura y puesta en crisis del pensamiento moderno.

\section{El teatro de Eduardo Calsamiglia: aproximaciones al teatro fantástico}

De Eduardo Calsamiglia conocemos hoy diez piezas dramáticas, aunque Abelardo Bonilla (1981, p. 203) afirma que existen seis obras más, inéditas, de las cuales fueron estrenadas unas cuantas. De su producción literaria la crítica ha dicho, principalmente, que se trata de un autor con influencias románticas y lo contrastan con los movimientos imperantes en 
ese momento, es decir, con el modernismo y el realismo. María Eugenia Dengo, en el prólogo a su Obra Literaria, dice que es un "personaje romántico y novelesco" y califica su estilo de "humorístico, satírico y filosófico" (Quesada-Soto y Lobo-Bejarano, 2006, pp. xvii-xix).

Por su parte, en un artículo que busca reivindicar la figura de Calsamiglia como un autor importante para nuestra historia literaria, titulado "Eduardo Calsamiglia: revalorización de un autor olvidado", Luis Gustavo Lobo (2002, p. 11) afirma que su obra es un "legado poético y teatral de corte netamente romántico en una época donde el movimiento literario vigente era el modernismo".

Además de estas, hay otras menciones a Calsamiglia en textos de Margarita Rojas y Flora Ovares (1995), compilaciones de Álvaro Quesada $(1993$; 2006) y en la Historia de la literatura costarricense de Abelardo Bonilla (1981). Los análisis se centran en el que parece ser su drama más conocido, llamado El Combate y que da título al volumen de piezas teatrales El combate y otras obras dramáticas, de 1914.

Es interesante la opinión de Bonilla respecto a sus textos dramáticos de los que afirma que "hay una curiosa mezcla de su sangre española, de su vocación, de sus estudios y de un ingenio brillante que trataba lo fantástico con igual desenvoltura que la vida nacional" (Bonilla, 1989, p. 203). Por desgracia, Bonilla no profundiza el comentario en lo referente a lo fantástico, no obstante, es evidente que tanto el mismo Calsamiglia -al calificar explícitamente Poderes invisibles de "drama fantástico"- como la crítica posterior reconoce una intención particular de la representación y del texto dramático, alejada de la versión más tradicional ya fuese extranjera o nacional. Bonilla concluye su referencia diciendo que Calsamiglia "No se concentró y no tuvo tiempo de realizar algo definitivo...pero dentro de lo relativo, fue un valor de época de nuestro teatro y, sin relación histórica o estética con nuestro medio, definió con acierto dos de sus personajes" (Bonilla, 1981, p. 204). ${ }^{1}$

Los comentarios de Bonilla nos confirman nuestra idea sobre ciertas piezas de Calsamiglia como teatro influenciado por las nuevas corrientes literarias europeas, la ciencia (sus estudios), así como por una intención estética específica y de ruptura con los cánones conocidos en la dramaturgia y el espectáculo costarricense de inicios del siglo XX.

De Poderes invisibles dice Bonilla que es un “drama simbólico”. Sobre ¡Él!, como ya se mencionó, no hay mayor comentario en las críticas literarias, más allá de las dos líneas de Abelardo Bonilla que la califican como un texto policiaco (Bonilla, 1981, p. 203).

En el ya mencionado prólogo a Bronces de antaño, Joaquín Vargas-Coto hace una breve referencia a otros textos dramáticos y en ella asocia la noción de fantástico con el sentido de "misterioso" y la presencia de personajes sobrenaturales, próxima a la concepción difundida por el teatro español del siglo XIX y principios del XX. Como se vio antes, en el caso de Abelardo Bonilla, el sentido del término fantástico queda en duda.

\footnotetext{
Y si sale tan bien librado de estas correrías por los campos humanos y terrestres, no de otro modo sale, cuando le llevan sus arrestos a meterse con lo divino e imaginario, escribiendo entonces, lindas comedias y fantásticos dramas, en los que entran personajes de alto fuste como son el Eterno Padre, el mismísimo y temido señor Mefistófeles con todos sus escuderos infernales, los diablillos cornudos y las diablescas, los ángeles y los serafines del reino de la dicha eterna, así como el viejo Pescador que hoy es portero celestial... Y allí están para probarlo El Diablo en el Cielo, Las opiniones de San Pedro y Poderes Invisibles... (Quesada-Soto y Lobo-Bejarano, 2006, p. 464)
}

En el drama en un acto Poderes invisibles, Calsamiglia parece hacer eco de la tradición del teatro español finisecular dieciochesco, primero al definirlo como "drama fantástico" y 
luego al hacer aparecer como personajes centrales a Lucifer y al arcángel Refulgente. Las pequeñas direcciones aportadas en las didascalias apuntan a una escenificación que pretende el asombro del espectador; como señalaba Matteo De Beni, se trata de lo fantástico visto como la búsqueda de un efecto, el texto en su "potencial espectacularidad" (De Beni, 2010, p. 54). Tan es así que estos personajes intervienen en las acciones de forma indirecta, esto es, que se supone que son invisibles y sus diálogos son tomados por el resto de personajes como sus propios pensamientos. Así lo estipula claramente Calsamiglia en la didascalia que abre la Escena III del Cuadro Segundo en la que dice que Lucifer "se supone invisible...pues solamente significa la representación fantástica del espíritu del mal" (Calsamiglia, 1914, p. 17).

Todo el primer cuadro se desarrolla en el infierno que se describe así: "Lucifer aparece sentado en un trono caprichoso de piedra negra, sobre el cual resalta lo rojo de su traje a la Mefistófeles. Un rayo azul lo hace destacarse sobre la escena" (Calsamiglia, 1914, p. 7). Más adelante, lo hará surgir "del fondo de la tierra, precedido por humo y llamas" (1914, p. 17). Otro aspecto que conecta este drama con el teatro fantástico decimonónico español es que en este cuadro no aparezcan personajes humanos vivos, pues la acción representa la llegada de las almas pecadoras escoltadas por los pecados capitales (que, como apunta De Beni [2010, p. 59], son "frecuentes en los dramas "fantásticos" del XIX y de principios del XX"). Completan la escena Astarote, la mano derecha de Lucifer y un grupo de diablos. Al final del cuadro, aparece Refulgente con un grupo de ángeles para retar a Lucifer a una lucha por el alma de Eunice.

En el segundo y último cuadro, se desarrolla la lucha entre ambas fuerzas por ganar las almas humanas para sus respectivos reinos, primero las de Ramón y Juanilla (un sacristán viejo y la criada de la casa) y, luego, la de Eunice quien se ve tentada a traicionar el amor del joven Rodrigo por las propuestas del Conde, un hombre rico quien ha enviado a su mujer a casa de sus padres y quiere, por medio de falsas promesas de matrimonio, riqueza y gloria, enamorar a Eunice y llevársela a Europa para después abandonarla. Finalmente, Eunice reconoce que ha "perdido el juicio, presa de poder extraño" (Calsamiglia, 1914, p. 47) y desiste del plan de huir con el Conde para quedarse con Rodrigo con lo que Refulgente triunfa sobre Lucifer y este tiene que regresar a lo profundo de la tierra.

Es evidente que, a pesar de que Calsamiglia juega en este drama con la idea del mal como inherente al ser humano (por ejemplo, Ramón, ya fuera del engaño de Lucifer, sigue pensando en el gesto impúdico de Juanilla de mostrarle su pie y el Conde no necesita de la ayuda infernal para mentir y perjurar), la que predomina y atraviesa el texto es la del mal como algo preternatural. El ser humano está sujeto a fuerzas mayores que lo inclinan a actuar bien o mal. La redención está supeditada a una lucha más allá de la voluntad humana que queda reducida a un principio de obediencia.

Por su parte, en ¡Él!, drama en tres actos, lo primero que llama la atención es que cada acto tenga un título, a saber: Acto primero "Un cuento de Conan Doyle", Acto segundo "El príncipe oscuro" y Acto tercero "Disnarda". Esto representa una ruptura con el modelo típico del texto dramático y lo acerca más bien a la narrativa. A cada una de estas partes corresponde un cambio textual tanto formal como de contenido y marca también la transición del relato fantástico a algo cercano a lo detectivesco.

En "Un cuento de Conan Doyle", Calsamiglia presenta un misterio y una historia ubicada en lo que parece ser un presente que luego veremos que se trata del pasado. Este primer acto coincide con la formulación del texto fantástico, pues en él el conflicto -la muerte del Conde Julio Foscari ejecutada por una mano momificada que este tenía encadenada en 
una urna- no parece tener una explicación racional. Los personajes, y nosotros como lectores, entramos en un estado de extrañamiento propio de lo fantástico y la duda sobre lo ocurrido se mantiene hasta el final del acto. El personaje del Conde es presentado como extraño, con reminiscencias a figuras tan clásicas de lo fantástico y del horror como Drácula; además, le reclama al personaje principal que no crea la historia que le cuenta: "[...] no puede comprender que existen en el mundo cosas inexplicables y sobrenaturales!" (Calsamiglia, 1914, p. 61). Los discursos religiosos, supersticiosos, etc., son constantemente enfrentados por el discurso médico, el científico y el del descreimiento de Carlos Story, el personaje que revelará "la verdad" al final de la obra.

En el segundo acto "El príncipe oscuro", el conflicto abandona el terreno de incógnita propio de lo fantástico, para transformarse en una representación de lo extraño, esto es, el conflicto y el acontecimiento que resultaba inexplicable en el acto anterior, encuentra en este acto una explicación mediante los medios de la razón y el estudio sistemático que hace Story de la filosofía y otros saberes, dice Story: "Soy investigador incorregible...Los secretos de los faquires. Allí nació la teosofía...esa ciencia es digna de estudio" (Calsamiglia, 1914, p. 75). Este acto es también particular, pues la historia se vuelve parte de sí misma, es decir, se acude a la metateatralidad como recurso, pues al mencionar el pueblo donde se desarrolló el primer acto se dice: “¿No fue allí donde sucedió aquella misteriosa aventura de la mano de momia de la cual tanto se ocuparon todos los periódicos del mundo?" (Calsamiglia, 1914, p. 75). Una de los personajes, Cristina, afirma “iEra la mano del diablo!”, a lo que Story replica: "No: era la mano de la venganza" (Calsamiglia, 1914, p. 76). El acto cierra con la promesa de Story de contarles a todos los personajes el final y develar el misterio de "la mano de momia".

El tercer acto, abandona pues lo extraño, para resolver por completo el misterio, y será precisamente Carlos Story quien devele toda la verdad. Este acto tiene tintes en efecto detectivescos, que han de haber hecho que Abelardo Bonilla la clasificara como un texto policiaco. Eso estaba anunciado desde el inicio con la mención a Arthur Conan Doyle, no obstante, el acto tiene elementos que lo relacionan con el texto de horror, por ejemplo, el topos convencional de la gente reunida en "corro o ronda" para escuchar un cuento de horror. Esto se concreta de forma particular, pues al mismo tiempo que escuchan la verdad, Disnarda descubre a su vez una verdad y mata al príncipe oscuro, o mejor dicho, a Simaritas el Manco.

El nombre del que resulta ser el personaje principal, Carlos Story, no es para nada gratuito, pues será mediante la historia o para ser precisos los "relatos" (y por eso mismo la palabra inglesa resulta perfecta) que se haga la transición entre lo fantástico, lo extraño y el horror detectivesco. Es el cuento el que permitirá el paso de la superstición y lo aparentemente mágico, a lo cognoscible y explicable mediante la ciencia, la investigación y el discurso racional.

¡Él! es un texto denso, hay múltiples historias entremezcladas (El Conde y Simaritas, Simaritas y Disnarda, Simaritas como El Príncipe y Sofía-Fernando y Story como elemento unificador de todas), múltiples tiempos (el pasado remoto que relaciona a Foscari con Simaritas y a este con Disnarda, el pasado más reciente con la muerte de Foscari y el viaje de Story a la India, y los presentes del baile en palacio y la narración), múltiples espacios (Maranto, el pueblo donde muere Foscari; la India, origen de la historia y lugar del viaje de Story; el palacio que por su descripción parece estar situado en Europa, donde se descubre el misterio), múltiples discursos (el religioso, el filosófico, el esotérico, el médico-forense, el astronómico, policiaco, etc.), es -en suma- un texto cargado de elementos simbólicos y que, además, plantea rupturas formales del modelo teatral en general y de la dramaturgia costarricense en particular. Calsamiglia supo combinar aspectos narrativos y dramáticos, metateatrales, metaficcionales e intertextuales, así 
como espacios, tiempos y modos literarios para crear una obra única en la literatura de este país.

La noción del mal es completamente distinta a la que planteaba Poderes invisibles o en El Combate, pues en ;Él! nada tienen que ver las fuerzas superiores, lo divino y lo infernal, cualquier concepción religiosa particular o, como en El Combate, en la que el mal se presenta como tensión bajo la forma de lo que Kant define como heteronomía, es decir, "el hecho de dar a su acción [a la humana] máximas diferentes de las de la razón misma" (Sichère, 1997, p. 180). Más bien, en el personaje de Simaritas, el Manco/Príncipe oscuro, cuyo verdadero nombre nunca sabemos y por ello representa a la vez a uno y a todos los sujetos, el mal es parte esencial de lo humano y está expresado en el deseo de venganza de Simaritas, pero también en el sistema que lo hace esclavo y que lo castiga por su robo, cortándole la mano. Es lo que la filosofía kantiana define como el mal radical (Sichère, 1995, p. 191), esto es, el mal como producto de un acto libre de nuestro albedrío y racionalidad.

Recapitulando podemos concluir respecto al teatro de principios del siglo XX y las obras de Eduardo Calsamiglia lo siguiente:

a. El final del siglo XIX y los primeros años del XX representan un período de auge del fenómeno teatral como espectáculo, en el que la influencia del teatro español es indudable y predominante en el gusto del público.

b. La noción de fantástico no pasó inadvertida tanto para el escritor como para su crítica (Vargas-Coto en 1919 y luego Bonilla en los cincuenta), eso sí, hay que reconocer las vicisitudes del término con el fin de no incurrir en anacronismos teórico-críticos.

c. Hay en el drama Poderes invisibles, referencias claras a la tradición española del teatro "fantástico" decimonónico y sus características particulares, muy distantes del fantástico moderno (entendido este como reacción al pensamiento racional de la Modernidad).

d. Por su parte, el drama iÉl! representa una ruptura estética, no solo con los cánones teatrales de lo fantástico español, sino con la estructura dramática en general, en una suerte de híbrido genérico entre la narrativa y el drama; y modal al hacer, además, un recorrido por distintas manifestaciones de lo fantástico, lo extraño y lo policiaco.

e. La reflexión filosófica sobre el mal es central en los textos dramáticos de Eduardo Calsamiglia, desde el mal como noción absoluta y preternatural hasta la concepción kantiana del mal radical.

f. El contexto sociocultural de producción de las piezas dramáticas de Calsamiglia juega un papel preponderante en su visión de la ciencia y la razón como medios para trascender la superstición y la superchería religiosa de la época.

En vista del breve recorrido anterior, se concluye que es urgente el estudio a profundidad de la obra dramática de Eduardo Calsamiglia, un autor, sin duda alguna, innovador y pionero del teatro y las letras costarricenses.

\section{Notas}

1. Ningún resaltado con negrita en las citas pertenece al texto original. 


\section{Bibliografía}

Alazraki, V. (2001). ¿Qué es lo neofantástico? Por D. Roas (Comp.). Teorías de lo fantástico. (265-282). Madrid: Arco/Libros, S.L.

Bonilla, A. (1981). Historia de la literatura costarricense. San José: Editorial STVDIVM.

Bravo, V. (1985). Los poderes de la ficción. Para una interpretación de la literatura fantástica. Caracas: Monte Ávila.

Callois, R. (1965). Au coeur du fantastique. París: Gallimard.

Calsamiglia, E. (1914). El Combate y otras obras dramáticas. San José: Imprenta Moderna.

Campra, R. (2008). Territorios de la ficción. Lo fantástico. España: Renacimiento.

Checa, J. (2009). Lo fantástico y el teatro español del siglo XX. Conferencia pronunciada en el Aula Magna. (152-177). Universidad Carlos III de Madrid. http://e-archivo.uc3m.es/ [Consulta mayo de 2013].

De Beni, M. (2010). Para una historia de la palabra "fantástico" en España. Iberoamérica Global. 3 (2), 46-67. http://dialnet.unirioja.es/ [Consulta mayo de 2013].

Díaz, R. (2005). Los costarricenses no han dejado de admirar las magnificencias del cielo: la evolución de las ideas astronómicas en Costa Rica 1814-1910. Diálogos Revista electrónica de historia. 6 (1), 282-307. http://www.historia.fcs.ucr.ac.cr/ [Consulta mayo de 2013].

Fumero, P. (2007). Los caminos de la dramaturgia costarricense. Escena. 61 (2), 85-90. http:// www.revistas.ucr.ac.cr/ [Consulta mayo de 2013].

Lobo, L. (2002). Eduardo Calsamiglia: revalorización de un autor olvidado [Resumen]. Revista Comunicación. 2 (22), 11-22. [Edición en Memoria del VIII Congreso de Filología, Lingüística y Literatura “Carmen Naranjo”, TEC].

Ovares, F. (2008). Modernidad y literatura en Costa Rica. Por J.A. Funes (Coord.). Antología literaria centroamericana: "El modernismo". (57-71). San José: Coordinación Educativa y Cultural Centroamericana, Imprenta Nacional. http://repositorio.una.ac.cr [Consulta junio de 2013].

Quesada, Á., Ovares, F., Rojas, M. y Santander, C. (1993). Antología del teatro costarricense, 1890-1950. San José: Editorial de la Universidad de Costa Rica.

Quesada-Soto, Á. (1995). La formación de la narrativa nacional costarricense (1890-1910). Enfoque histórico social. San José: Editorial de la Universidad de Costa Rica.

Quesada-Soto, Á. y L.G. Lobo-Bejarano. (Comps.). (2006). Obra literaria de Eduardo Calsamiglia. San José: Editorial de la Universidad de Costa Rica.

Ramírez, J. (1994). La nueva esfera de lo sagrado en la ideología patriarcal en El Combate de Eduardo Calsamiglia. Escena. 16 (32-33), 21-25. http://www.repositorio.una.ac.cr/ [Consulta mayo de 2013].

Roas, D. (Comp.). (2001). Teorías de lo fantástico. Madrid: ARCO/LIBROS.

Roas, D. (2009). Lo fantástico como desestabilización de lo real: elementos para una definición. Por T. López-Pellisa y F.Á. Moreno-Serrano (Eds.). Ensayos sobre ciencia ficción y literatura fantástica: Actas del Primer Congreso Internacional de literatura fantástica y ciencia ficción. (94-120). Universidad Carlos III de Madrid. http://earchivo.uc3m.es/ [Consulta mayo de 2013]. 
Rojas, M. y Ovares, F. (1995). 100 años de literatura costarricense. San José: Ediciones FARBEN.

Rubio, J. (1989). Modernismo y teatro de ensueño. Anales de la literatura española contemporánea. 14 (1-3), 199-222. JSTOR. [Consulta mayo de 2013].

Sandoval, V. (1987). Dramaturgia costarricense. Revista Iberoamericana. 53 (138-139), 173 192. http://revista-iberoamericana.pitt.edu/ [Consulta mayo de 2013].

Sichère, B. (1995). Historias del mal. (A.L. Bixio, tr.). Barcelona: Editorial Gedisa.

Todorov, T. (2006). Introducción a la literatura fantástica. Buenos Aires: Paidós.

Trancón, S. (2006). Teoría del teatro: Bases para el análisis de la obra dramática. Madrid: Fundamentos.

Trouillhet-Manso, J. (2009). El teatro fantástico de comienzos del siglo XX: el caso de ValleInclán. Por T. López-Pellisa y F.Á. Moreno-Serrano (Eds.). Ensayos sobre ciencia ficción y literatura fantástica: actas del Primer Congreso Internacional de literatura fantástica y ciencia ficción. (794-803). Universidad Carlos III de Madrid. http://earchivo.uc3m.es/ [Consulta mayo de 2013].

Vax, L. (1973). Arte y literatura fantástica. Buenos Aires: Eudeba. 
